\title{
Perfil de resistência microbiana das infecções relacionadas à assistência à saúde de um hospital de doenças infectocontagiosas de uma capital do Nordeste brasileiro
}

Microbial resistance profile of infections related to health care in a hospital for infectious diseases

in a capital of Northeastern Brazil

Perfil de resistencia microbiana de infecciones relacionadas con la atención de la salud en un hospital de enfermedades infecciosas de una capital del Noreste de Brasil Universidade Federal de Alagoas, Brasil E-mail: karen.borges@eenf.ufal.br

Karine Rocha Borges

ORCID: https://orcid.org/0000-0001-7991-0290 Universidade Federal de Alagoas, Brasil

E-mail: karine.krb@gmail.com

Adriana Viera Junges

ORCID: https://orcid.org/0000-0002-7734-0754

\begin{abstract}
Resumo
Introdução: as Infecções Relacionadas à Assistência à Saúde são tidas como um importante agravo à saúde, por resultar em alta mortalidade, prolongamento da hospitalização, aumento do custo da assistência e favorecimento da seleção e disseminação de micro-organismos multirresistentes. Objetivo: descrever o perfil de resistência microbiana das infecções relacionadas à assistência à saúde de um hospital de doenças infectocontagiosas de uma capital do nordeste brasileiro. Métodos: estudo observacional descritivo, quantitativo e retrospectivo. Pesquisa realizada por meio de coleta de dados no setor de comissão de controle de infecção hospitalar nas fichas de notificações, exames de cultura e teste de sensibilidade antimicrobiana dos pacientes que foram internados nos anos de 2018 e 2019 e desenvolveram infecção durante o período de internação. Resultados: foram analisadas 52 fichas de notificações de infecções relacionadas à assistência à saúde. Identificaram-se seis sítios de infecção: pulmão $(26,0)$, trato urinário $(13,0)$, tegumento $(6,0)$, traqueia $(3,0)$, intra-abdominal $(2,0)$ e sangue $(2,0)$; com destaque para o sistema respiratório: infecções pulmonares foram observadas em 50,0\% dos indivíduos. Em nossa pesquisa a maior frequência de resistência observada nas enfermarias foi ceftriaxona (11,0/84,6\%), seguida de sulfazotrim $(5,0 / 83,3 \%)$ e ampicilina com sulbactam $(8,0 / 80,0 \%)$. Enquanto na Unidade de Terapia Intensiva, o maior perfil de resistência foi sulfazotrim $(4,0 / 100 \%)$, seguido da ampicilina com sulbactam $(9,0 / 90,0 \%)$, piperacilina com tazabactam sódico $(9,0 / 75,0 \%)$. Conclusão: os dados obtidos mostram elevada frequência de multirresistência principalmente em bactérias Gramnegativas: Pseudomonas aeruginosa (8,0), seguido de Acinetobacter baumannii $(7,0)$, e da Escherichia gergoviae $(5,0)$.
\end{abstract}

Palavras-chave: Enfermagem; Infecção hospitalar; Resistência microbiana a medicamentos. 


\begin{abstract}
Introduction: health Care-Related Infections are seen as an important health problem, as they result in high mortality, prolonged hospitalization, increased cost of care and favored the selection and dissemination of multi-resistant microorganisms. Objective: to describe the profile of microbial resistance of diseases related to health care in a hospital for infectious diseases in a capital of northeastern Brazil. Methods: descriptive, quantitative and retrospective observational study. Research carried out through data collection in the hospital infection control commission sector in the notification forms, culture tests and antimicrobial sensitivity test of patients who were hospitalized in the years 2018 and 2019 and developed infection during the hospitalization period. Results: 52 forms of notifications of infections related to health care were analyzed. Six infection sites were identified: lung (26.0), urinary tract (13.0), integument (6.0), trachea (3.0), intra-abdominal (2.0) and blood (2,0); with emphasis on the respiratory system: pulmonary diseases were observed in $50.0 \%$ of those desired. In our research, the highest frequency of resistance observed in the wards of ceftriaxone (11.0/84.6\%), followed by sulfazotrim (5.0/83.3\%) and ampicillin with sulbactam (8.0/80.0\%) While in the Intensive Care Unit, the highest resistance profile was sulfazotrim (4.0/100\%), followed by ampicillin with sulbactam $(9.0 / 90.0 \%)$, piperacillin with sodium tazabactam $(9.0 / 75.0 \%)$. Conclusion: the data presented show the frequency of multidrug resistance mainly in Gram-negative bacteria: Pseudomonas aeruginosa (8.0), followed by Acinetobacter baumannii (7.0), and Escherichia gergoviae (5.0).
\end{abstract}

Keywords: Nursing; Cross infection; Drug resistance, microbial.

\title{
Resumen
}

Introducción: las Infecciones Relacionadas con la Salud son vistas como un problema de salud importante, ya que resultan en alta mortalidad, hospitalización prolongada, mayor costo de la atención y favorecen la selección y diseminación de microorganismos multirresistentes. Objetivo: describir el perfil de resistencia microbiana de enfermedades relacionadas con la atención de la salud en un hospital de enfermedades infecciosas de una capital del noreste de Brasil. Métodos: estudio observacional descriptivo, cuantitativo y retrospectivo. Investigación realizada a través de recolección de datos en el sector de comisiones de control de infecciones hospitalarias en los formularios de notificación, pruebas de cultivo y prueba de sensibilidad antimicrobiana de pacientes que fueron hospitalizados en los años 2018 y 2019 y desarrollaron infección durante el período de internación. Resultados: se analizaron 52 formas de notificaciones de infecciones relacionadas con la atención de la salud. Se identificaron seis sitios de infección: pulmón $(26,0)$, tracto urinario $(13,0)$, tegumento $(6,0)$, tráquea $(3,0)$, intraabdominal $(2,0)$ y sangre $(2,0)$; con énfasis en el sistema respiratorio: se observaron enfermedades pulmonares en el 50,0\% de las deseadas. En nuestra investigación, la mayor frecuencia de resistencia observada en las salas de ceftriaxona $(11,0 / 84,6 \%)$, seguida de sulfazotrim $(5,0 / 83,3 \%)$ y ampicilina con sulbactam $(8,0 / 80,0 \%)$. El perfil de resistencia más alto fue sulfazotrim $(4.0 / 100 \%)$, seguido de ampicilina con sulbactam (9.0/90.0\%), piperacilina con tazabactam de sodio (9.0/75, 0\%). Conclusión: los datos presentados muestran la frecuencia de multirresistencia principalmente en bacterias Gram-negativas: Pseudomonas aeruginosa (8.0), seguida de Acinetobacter baumannii (7.0) y Escherichia gergoviae (5.0).

Palabras clave: Enfermería; Infección hospitalaria; Farmacorresistencia microbiana.

\section{Introdução}

As infecções relacionadas à assistência à saúde (IRAS) são consideradas eventos adversos e têm provocado um aumento da morbimortalidade de pacientes, afetando significativamente a saúde de indivíduos hospitalizados e ocasionando repercussões sociais e econômicas para as comunidades, os sistemas de saúde e os países (Araújo \& Pereira, 2017).

Dessa maneira, as IRAS têm causado uma grande preocupação nos hospitais brasileiros, em especial aquelas provocadas por micro-organismos multirresistentes, que são aqueles que desenvolvem resistência a no mínimo duas classes de antimicrobianos. Em consequência da multirresistência, as opções de tratamento tornam-se reduzidas, aumentando o tempo de hospitalizações, o número de óbitos e os custos assistenciais (Prates, Silva, Fernandes \& Cesar, 2020). Nesse contexto, verifica-se o aumento das IRAS nos serviços de saúde brasileiro, implicando no uso de diversos fármacos da classe dos antibióticos em amplo espectro, quando usado de forma indiscriminada, isto possibilita a ocorrência da resistência microbiana no Brasil e no mundo (Ferreira, Azevedo, Salvador, Morais, Paiva \& Santos, 2019).

A resistência a antimicrobianos (RAM) está relacionada a vários fatores, entre eles, o mau uso de medicamentos antimicrobianos, programas inadequados ou inexistentes de prevenção e controle de infecções. Nesse sentido, o manejo para o controle e disseminação de micro-organismos resistentes aos antimicrobianos e o crescimento de bactérias com formas novas de resistência implicam numa conduta mais efetiva, através do estabelecimento de estratégias nacionais, que enfatizem a detecção, prevenção e redução da resistência microbiana nos serviços de saúde do país (World Health Organization, 2015). 
Dessa forma, destaca-se a efetivação de medidas de cuidados aos pacientes, como barreiras entre indivíduos infectados/colonizados e higienização frequente das mãos. Além disso, é de fundamental importância a existência de um laboratório de microbiologia preparado para identificar o mais breve possível uma infecção, apresentando o perfil de resistência aos antibióticos de forma eficaz e rápida, para que seja possível realizar a conduta correta por parte dos profissionais de saúde (Nere, Silva, Silva, Rodrigues, Pereira \& Soares, 2017).

Nessa perspectiva, este estudo tem como objetivo descrever o perfil de resistência microbiana das infecções relacionadas à assistência à saúde de um hospital de doenças infectocontagiosa de uma capital do Nordeste.

\section{Metodologia}

Trata-se de um estudo observacional de abordagem quantitativa do tipo retrospectivo. O local do estudo foi um hospital de doenças infectocontagiosas de uma capital do nordeste brasileiro, no setor da comissão de controle de infecção hospitalar (CCIH).

Os critérios de elegibilidade para compor a amostra foram os seguintes: critérios de inclusão - fichas de notificação de IRAS de pacientes que foram internados no período de janeiro de 2018 a dezembro de 2019 e desenvolveram infecção durante o período de internação; critério de exclusão - foram excluídas as fichas de notificação de IRAS que apresentaram campos fundamentais sem o devido preenchimento, como os micro-organismos isolados, sítio de infecção e resultados dos antibiogramas. Com base nesses critérios, foram analisadas um total de 73 fichas de notificações de IRAS dos pacientes que foram internados no período de janeiro de 2018 a dezembro de 2019, dessas 21 foram descartadas da amostra por apresentarem informações incompletas, restando um total de 52 fichas que compuseram a amostra.

Nas fichas de notificações de IRAS dos pacientes, assim como nos exames de cultura e teste de sensibilidade antimicrobiana, foram coletados os dados para levantamento do perfil de resistência e sensibilidade dos antimicrobianos referentes às seguintes informações/variáveis: micro-organismos sensíveis, intermediários ou resistentes aos antibióticos; tipos de micro-organismos isolados; sítio de infecção; material biológico da análise; caracterização do paciente quanto ao sexo, idade, diagnóstico clínico que motivou o internamento e local de internação (enfermaria ou Unidade de Terapia Intensiva).

Os dados coletados foram organizados e armazenados em uma planilha idealizada para essa finalidade, usando o programa Microsoft Excel @ (2019) e analisados no programa IBM Statistical Package for the Social Sciences (SPSS) na versão 21. Os resultados foram expressos pela estatística descritiva em medidas de posição (média, mediana, máximo), frequência absoluta e relativa, sendo apresentados por meio de tabelas, gráficos e quadros, no intuito de facilitar e organizar as informações obtidas.

$\mathrm{Na}$ análise estatística, as variáveis categóricas foram descritas em valores absolutos, percentuais e as contínuas por meio das medidas de tendência central e dispersão. $O$ teste de Kolmogorov-Smirnov $(p=0,200)$ foi utilizado para a avaliação da normalidade dos dados. Uma vez confirmada, foi utilizado o teste T-Student para comparação das variáveis contínuas.

O presente estudo foi aprovado pelo Comitê de Ética em pesquisa de uma capital do nordeste brasileiro sob CAAE: 28676720.3.0000.5013 e parecer: 3.890.458, respeitando os princípios e diretrizes contidos na Resolução CNS nº 466/12 e 563/2017 do Conselho Nacional de Pesquisa em Saúde que envolve seres humanos.

\section{Resultados}

Foram analisados dados de 73 pacientes hospitalizados, porém 21 foram descartadas por não atenderem aos critérios de inclusão, dessa forma foram utilizados na análise 52 fichas de notificações de IRAS dos internados entre janeiro de 2018 a dezembro de 2019. A partir dessas fichas, dos pacientes analisados $36(69,2 \%)$ eram do sexo masculino e 16 (30,8\%) do sexo feminino. A média de idade foi 44,36 ( $\pm 13,6)$, sem diferença significativa entre os sexos ( $p=0,463)$, sendo 43,65 $\pm 11,38$ para o 
sexo masculino e 47,00 $\pm 17,18$ para o sexo feminino (Figura 1).

Figura 1 - Comparação entre as variáveis, idade e sexo dos pacientes incluídos no estudo de um hospital de doenças infectocontagiosas de uma capital da região Nordeste. Brasil, 2021 ( $\mathrm{n}=52)$.

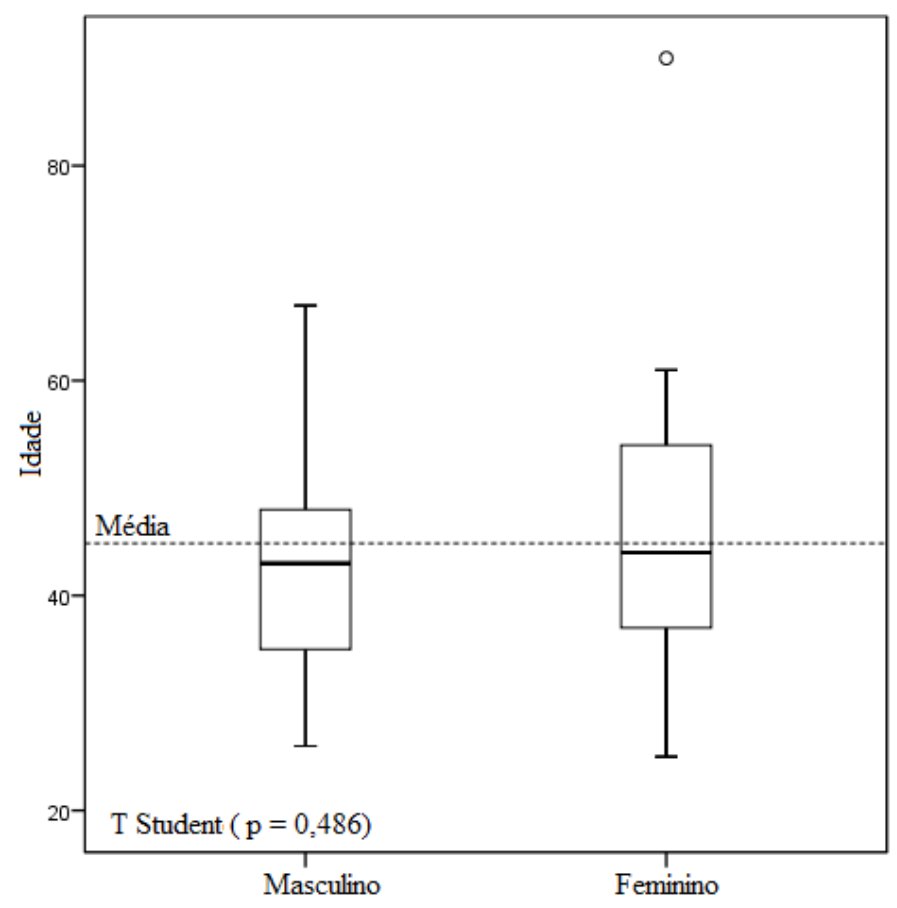

Fonte: Autores (2021).

Dos indivíduos que desenvolveram infecção durante a internação, 59,6\% (n=31) estavam internados nas enfermarias e 40,4\% ( $\mathrm{n}=21)$ na UTI. O percentual de homens internados na UTI foi 1,88 vezes maior do que o percentual de mulheres (47,2\% dos homens e 25,0\% nas mulheres). A tabela 1 apresenta as varáveis: sexo, sítio de infecção e número de diagnósticos clínicos nas enfermarias e na Unidade de Terapia Intensiva (UTI) e seus respectivos percentuais conforme os dados coletado e tabulado na pesquisa. Dessa forma, foram identificados seis sítios de infecção, com destaque para o sistema respiratório: infecções pulmonares foram observadas em $50 \%$ dos indivíduos. Percentual de $38,5 \%(\mathrm{n}=20)$ dos pacientes apresentavam três ou mais diagnósticos clínicos (Tabela 1). 
Tabela 1 - Caracterização epidemiológica dos pacientes internados nas enfermarias e UTI, no período de janeiro de 2018 a dezembro de 2019 de um hospital de doenças infectocontagiosas de uma capital do Nordeste. Brasil, 2021.

\begin{tabular}{lllllll}
\hline & \multicolumn{2}{l}{ Enfermarias } & UTI & \multicolumn{3}{c}{ Total } \\
\cline { 2 - 6 } Variável & $\mathrm{N}$ & $\%$ & $\mathrm{~N}$ & $\%$ & $\mathrm{~N}$ & $\%$ \\
\hline Sexo & & & & & & \\
Masculino & 19 & 52,8 & 17 & 47,2 & 36 & 69,2 \\
Feminino & 12 & 75,0 & 4 & 25,0 & 16 & 30,8 \\
Sítio de Infecção & & & & & \\
Traqueia & 1 & 33,3 & 2 & 66,7 & 3 & 5,8 \\
Trato Urinário & 10 & 76,9 & 3 & 23,1 & 13 & 25,0 \\
Tegumento (Pele) & 5 & 83,3 & 1 & 16,7 & 6 & 11,6 \\
Pulmão & 11 & 42,3 & 15 & 57,7 & 26 & 50,0 \\
Sangue & 2 & 100,0 & 0 & 0,0 & 2 & 3,8 \\
Intra-abdominal & 2 & 100,0 & 0 & 0,0 & 2 & 3,8 \\
No de Diagnósticos & & & & & & \\
Clínicos & & & & & & \\
Um & 8 & 57,2 & 6 & 42,8 & 14 & 26,9 \\
Dois & 13 & 72,2 & 5 & 27,8 & 18 & 34,6 \\
Três ou mais & 10 & 50,0 & 10 & 50,0 & 20 & 38,5 \\
\hline Total & $\mathbf{3 1}$ & $\mathbf{5 9 , 6 \%}$ & $\mathbf{2 1}$ & $\mathbf{4 0 , 4 \%}$ & $\mathbf{5 2}$ & $\mathbf{1 0 0 , 0 \%}$ \\
\hline
\end{tabular}

Fonte: Autores (2021).

Quanto ao diagnóstico clínico que motivou a internação, observou-se predomínio de internações pela Síndrome da Imunodeficiência Adquirida - SIDA (42,9\% dos internados na enfermaria e 41,9\% dos internados na UTI), seguido de neurotoxoplasmose $(19,4 \%$ e $19,0 \%$, respectivamente) e Tuberculose (19,4\% e 19,0\%, respectivamente) (Figura 2). 
Figura 2 - Prevalência de diagnóstico clínico, conforme local de internamento de um hospital de doenças infectocontagiosas do Nordeste. Brasil, 2021.

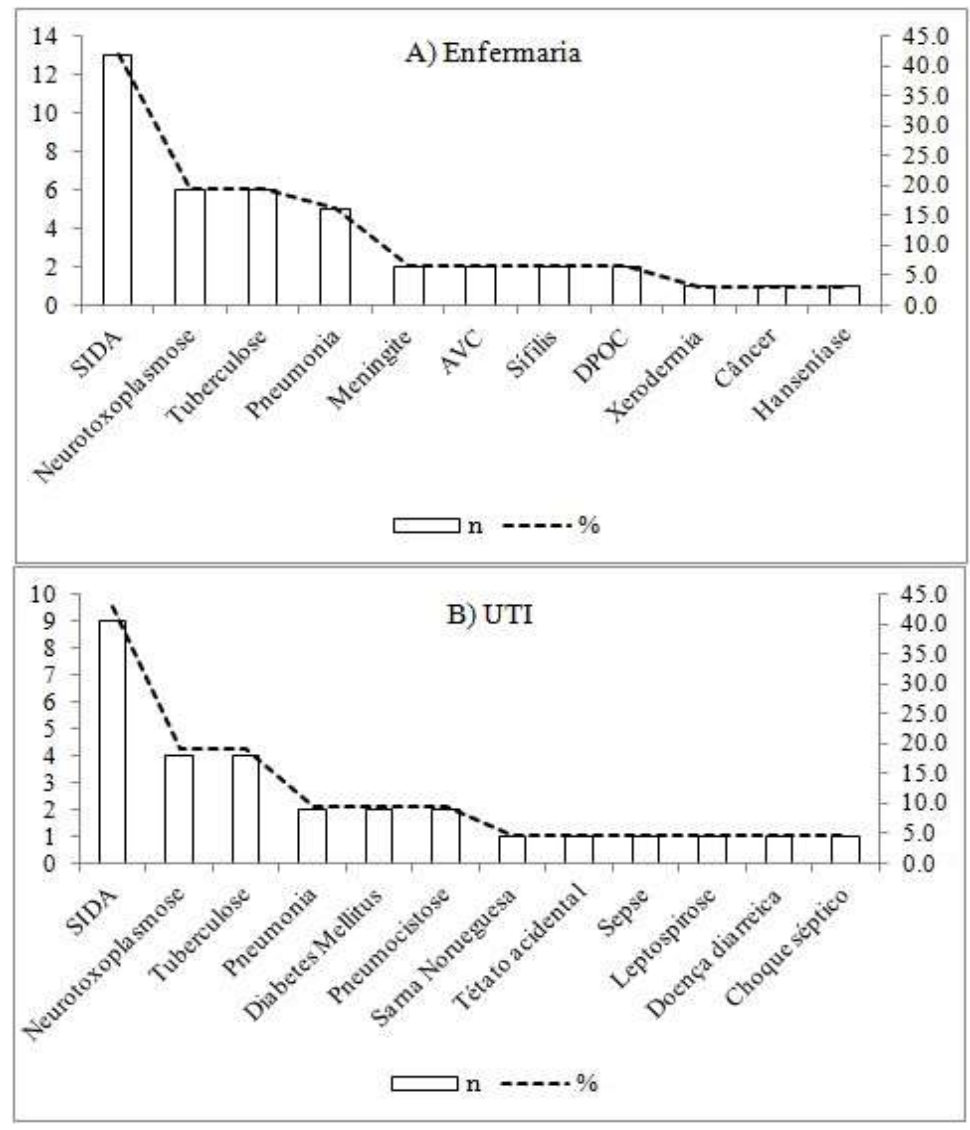

Fonte: Autores (2021).

O material coletado para a análise do antibiograma foi em sua maioria a secreção traqueal, sendo $41,4 \%$ dos pacientes internados na enfermaria e 58,6\% internados na UTI. Nesse estudo, os micro-organismos mais comumente isolados nas enfermarias foram os gram-negativos: Pseudomonas aeruginosa (61,5\%), seguido por Escherichia gergoviae (55,6\%) e Acinetobacter baumannii $(53,8 \%)$. Os micro-organismos mais isolados na UTI foram os Gram-negativos: Acinetobacter baumannii (46,2\%), seguidos da Escherichia gergoviae (44,4\%) e da Pseudomonas aeruginosa (38,5\%). Além disso, a proporção de pacientes que são sensíveis a quatro ou mais drogas é 1,89\% vezes maior na enfermaria do que na UTI (65,4 e 34,6 respectivamente); enquanto a proporção de pacientes que são resistentes a quatro ou mais drogas é 1,26\% vezes maior na enfermaria do que na UTI (57,6 e 42,4 respectivamente). Estas informações estão apresentadas detalhadamente na Tabela 2. 
Tabela 2 - Descrição dos materiais biológicos coletados para análises de cultura, micro-organismos isolados e o perfil de resistência e sensibilidade antimicrobiana de um hospital de doenças infectocontagiosas do Nordeste. Brasil, 2021.

\begin{tabular}{|c|c|c|c|c|c|c|}
\hline \multirow{2}{*}{ Variável } & \multicolumn{2}{|c|}{ Enfermaria } & \multicolumn{2}{|c|}{ UTI } & \multicolumn{2}{|c|}{ Total } \\
\hline & $\mathrm{N}$ & $\%$ & $\mathrm{~N}$ & $\%$ & $\mathrm{~N}$ & $\%$ \\
\hline \multicolumn{7}{|l|}{ Material Biológico } \\
\hline Aspirado Traqueal & 12 & 41,4 & 17 & 58,6 & 29 & 55,8 \\
\hline Urina & 10 & 76,9 & 3 & 23,1 & 13 & 25,0 \\
\hline Ponta do Cateter & 1 & 100,0 & 0 & 0,0 & 1 & 1,9 \\
\hline Secreção de Ferida & 5 & 83,3 & 1 & 16,7 & 6 & 11,5 \\
\hline Punção Abdominal & 1 & 100,0 & 0 & 0,0 & 1 & 1,9 \\
\hline Sangue & 2 & 100,0 & 0 & 0,0 & 2 & 3,8 \\
\hline \multicolumn{7}{|l|}{ Agente Identificado } \\
\hline Klebsiella aerogenes & 1 & 50,0 & 1 & 50,0 & 2 & 3,8 \\
\hline Sthaphylococcus aureus & 3 & 100,0 & 0 & 0,0 & 3 & 5,8 \\
\hline Acinetobacter baumannii & 7 & 53,8 & 6 & 46,2 & 13 & 25,0 \\
\hline Pseudomonas aeruginosa & 8 & 61,5 & 5 & 38,5 & 13 & 25,0 \\
\hline Escherichia coli & 5 & 100,0 & 0 & 0,0 & 5 & 9,7 \\
\hline Escherichia gergoviae & 5 & 55,6 & 4 & 44,4 & 9 & 17,3 \\
\hline Enterobacter sp. & 1 & 100,0 & 0 & 0,0 & 1 & 1,9 \\
\hline Klebsiella oxytoca & 0 & 0,0 & 1 & 100,0 & 1 & 1,9 \\
\hline Klebsiella pneumoniae & 1 & 20,0 & 4 & 80,0 & 5 & 9,6 \\
\hline \multicolumn{7}{|c|}{ Sensibilidade aos Fármacos } \\
\hline Zero & 5 & 50,0 & 5 & 50,0 & 10 & 19,2 \\
\hline Uma a três & 9 & 56,2 & 7 & 43,8 & 16 & 30,8 \\
\hline Quatro ou mais & 17 & 65,4 & 9 & 34,6 & 26 & 50,0 \\
\hline \multicolumn{7}{|l|}{ Resistência aos Fármacos } \\
\hline Zero & 5 & 62,5 & 3 & 37,5 & 8 & 15,4 \\
\hline Uma a três & 7 & 63,6 & 4 & 36,4 & 11 & 21,2 \\
\hline Quatro ou mais & 19 & 57,6 & 14 & 42,4 & 33 & 63,5 \\
\hline Medidas Quantitativas & \multicolumn{2}{|c|}{ Média $\pm D P$} & \multicolumn{2}{|c|}{ Média $\pm D P$} & \multicolumn{2}{|c|}{ Teste T Studen } \\
\hline $\mathrm{N}^{\mathrm{o}}$ de drogas sensíveis & \multicolumn{2}{|c|}{$4,0 \pm 2.8$} & \multicolumn{2}{|c|}{$3,5 \pm 3,3$} & \multicolumn{2}{|c|}{0,539} \\
\hline $\mathrm{N}^{\mathrm{o}}$ de drogas resistentes & \multicolumn{2}{|c|}{$4,2 \pm 3.3$} & \multicolumn{2}{|c|}{$5,6 \pm 3,9$} & \multicolumn{2}{|c|}{0,139} \\
\hline
\end{tabular}

Fonte: Autores (2021).

A Tabela 3 apresenta o percentual de resistência dos micro-organismos aos antibióticos isolados nas culturas dos pacientes hospitalizados nas enfermarias e UTI. Esta tabela apresenta ainda a classificação dos antibióticos, as drogas que foram testadas e a resistência. Nesta perspectiva, destacam-se os antibióticos com maior resistência nas enfermarias: ceftriaxona - cefalosporina de $3^{\mathrm{a}}$ geração $(84,6 \%)$, seguido sulfazotrim - sulfonamida $(83,3 \%)$ e ampicilina com subactam Penicilina -Inibidor da Beta-Lactamase (80,0\%). Enquanto na UTI foram: sulfazotrim - Sulfonamida (100,0\%), seguido por ampicilina com subactam - Penicilina -Inibidor da Beta-Lactamase (90\%) e piperacilina com tazobactam sódico - Penicilina (75,0\%). 
Tabela 3 - Perfil de resistência antimicrobiana aos antibióticos dos micro-organismos isolados nas culturas de acordo com o antibiograma de um hospital de doenças infectocontagiosas de uma capital do Nordeste. Brasil, 2021.

\begin{tabular}{|c|c|c|c|c|c|c|c|}
\hline & & Enferm & $i a(n=31)$ & $U T$ & $n=21)$ & Tota & $n=52)$ \\
\hline \multirow{2}{*}{$\begin{array}{l}\text { Classificação do } \\
\text { Antibiótico }\end{array}$} & Antimicrobianos & Testados & Resistência & Testados & Resistência & Testados & Resistência \\
\hline & & $\mathrm{n}(\%)$ & n (\%) & $\mathrm{n}(\%)$ & n (\%) & $\mathrm{n}(\%)$ & $\mathrm{n}(\%)$ \\
\hline \multirow[b]{2}{*}{ Aminoglicosídeos } & Amicacina & $17(54,8)$ & $6(35,3)$ & $8(38,1)$ & $5(62,5)$ & $25(48,07)$ & $11(21,15)$ \\
\hline & Gentamicina & $14(45,2)$ & $8(57,1)$ & $10(47,6)$ & $6(60,0)$ & $24(46,15)$ & $14(26,92)$ \\
\hline \multirow{3}{*}{ Carbapenêmicos } & Ertapenem & $18(58,1)$ & $8(44,4)$ & $16(76,2)$ & $10(62,5)$ & $34(65,38)$ & $18(34,61)$ \\
\hline & Imipenem & $25(80,6)$ & $9(36,0)$ & $17(80,9)$ & $8(47,1)$ & $42(80,76)$ & $17(32,69)$ \\
\hline & Meropenem & $19(61,3)$ & $6(31,6)$ & $18(85,7)$ & $9(50,0)$ & $37(71,15)$ & $15(28,84)$ \\
\hline $\begin{array}{c}\text { Cefalosporina } 2^{a} \\
\text { Geração }\end{array}$ & Cefuroxina & $4(12,9)$ & $1(25,0)$ & $3(14.3)$ & $2(66,6)$ & $7(13,46)$ & $3(5,76)$ \\
\hline \multirow{3}{*}{$\begin{array}{c}\text { Cefalosporina } 3^{a} \\
\text { Geração }\end{array}$} & Cefotaxima & - & - & $2(9,6)$ & $1(50,0)$ & $2(3,84)$ & $1(1,92)$ \\
\hline & Ceftazidima & $13(41,9)$ & $8(61,5)$ & $7(33,3)$ & $4(57,1)$ & $20(38,46)$ & $12(60,0)$ \\
\hline & Ceftriaxona & $13(41,9)$ & $11(84,6)$ & $11(52,4)$ & $8(72,7)$ & $24(46,15)$ & $19(36,53)$ \\
\hline $\begin{array}{c}\text { Cefalosporina } 4^{a} \\
\text { Geração }\end{array}$ & Cefepime & $19(61,3)$ & $11(57,9)$ & $15(71,4)$ & $9(60,0)$ & $34(65,38)$ & $20(38,46)$ \\
\hline Fosfomicina & Fosfomicina & $2(6,4)$ & - & - & - & $2(3,84)$ & - \\
\hline \multirow{2}{*}{ Glicopeptídeos } & Teicoplanina & $3(9,6)$ & - & - & - & $3(5,76)$ & - \\
\hline & Vancomicina & $1(3,2)$ & - & - & - & $1(1,92)$ & - \\
\hline Lincosamida & Clindamicina & $2(6,4)$ & $2(100,0)$ & - & - & $2(3,84)$ & $2(3,84)$ \\
\hline Macrolídeo & Eritromicina & $3(9,6)$ & $3(100,0)$ & - & - & $3(5,76)$ & $3(5,76)$ \\
\hline Monobactâmico & Aztreonam & $9(29,0)$ & $5(55,5)$ & $10(47,6)$ & $7(70,0)$ & $19(36,53)$ & $12(23,07)$ \\
\hline Oxazolidinonas & Linezulida & $3(9,6)$ & - & - & - & $3(5,76)$ & - \\
\hline \multirow{3}{*}{ Penicilinas } & Oxacilina & $1(3,2)$ & $1(100,0)$ & - & - & $1(1,92)$ & $1(1,92)$ \\
\hline & $\begin{array}{l}\text { Penicilina G. } \\
\text { Cristalina }\end{array}$ & $1(3,2)$ & - & - & - & $1(1,92)$ & - \\
\hline & $\begin{array}{l}\text { Piperacilina + } \\
\text { Tazobactam } \\
\text { Sódico }\end{array}$ & $20(64,5)$ & $7(35,0)$ & $12(57,1)$ & $9(75,0)$ & $32(61,53)$ & $16(30,76)$ \\
\hline \multirow{2}{*}{$\begin{array}{c}\text { Penicilina - } \\
\text { Inibidor da Beta- } \\
\text { Lactamase }\end{array}$} & $\begin{array}{l}\text { Amoxicilina + } \\
\text { Clavulanato de } \\
\text { Potássio }\end{array}$ & $5(16,1)$ & $3(60,0)$ & $5(23,8)$ & $2(40,0)$ & $10(19,23)$ & $5(9,61)$ \\
\hline & $\begin{array}{l}\text { Ampicilina } \\
+ \text { Sulbactam }\end{array}$ & $10(32,2)$ & $8(80,0)$ & $10(47,6)$ & $9(90,0)$ & $20(38,46)$ & $17(85,0)$ \\
\hline Polimixinas & Polimixina B & $4(12,9)$ & - & $1(4.8)$ & - & $5(9,61)$ & - \\
\hline \multirow{3}{*}{ Quinolonas } & Ciprofloxacino & $20(64,5)$ & $10(50,0)$ & $15(71,4)$ & $10(66,6)$ & $35(67,30)$ & $20(38,46)$ \\
\hline & Levofloxacino & $17(54,8)$ & $11(64,7)$ & $19(90,5)$ & $12(63,2)$ & $36(69,23)$ & $23(44,23)$ \\
\hline & Norfloxacino & $12(38,7)$ & $8(66,6)$ & $4(19,1)$ & $2(50,0)$ & $16(30,76)$ & $10(19,23)$ \\
\hline Sulfonamidas & Sulfazotrim & $6(19,3)$ & $5(83,3)$ & $4(19,1)$ & $4(100,0)$ & $10(19,23)$ & $9(17,30)$ \\
\hline \multirow{2}{*}{ Tetraciclinas } & Minocilina & - & - & $1(4,8)$ & $1(100,0)$ & $1(1,92)$ & $1(1,92)$ \\
\hline & Tetraciclina & $1(3,2)$ & $1(100,0)$ & - & - & $1(1,92)$ & $1(1,92)$ \\
\hline
\end{tabular}

Fonte: Autores (2021). 


\section{Discussão}

Evidenciou-se, no presente estudo, uma maior frequência de infecções do trato respiratório, seguida do trato urinário, causadas por microrganismos gram-negativos. Entre os microrganismos isolados houve uma maior taxa de resistência a fármacos da classe das Cefalosporinas de $3^{a}$ Geração, sulfazotrim e ampicilina com subactam. A maioria dos pacientes apresentou dois ou mais diagnósticos clínicos, sendo a SIDA, neurotoxoplasmose e tuberculose os mais frequentes, o que fragiliza o indivíduo e pode predispor as IRAS.

Em relação à caracterização dos participantes dessa pesquisa destaca-se uma hospitalização maior no gênero masculino (69,2\%), diferente do que foi encontrado na pesquisa de Araújo et al. (2018) no qual a predominância foi do gênero feminino (63,6\%), sendo esta realizada com o objetivo de determinar a prevalência de Infecção Relacionada à Assistência à Saúde (IRAS) em pacientes internados em uma UTI de um hospital universitário, referência em média e alta complexidade em diversas especialidades, localizado na cidade de Natal, no Estado do Rio Grande do Norte.

As infecções do trato respiratório foram as mais frequentes no presente estudo, sendo a coleta de aspirado traqueal de 55,8\%. Na pesquisa de Basso, Pulcinelli, Aquino e Santos (2016) o aspirado traqueal $(65,3 \%)$ apresentou maior frequência, o objetivo desse estudo foi identificar quais os micro-organismos prevalentes em unidades de terapia intensiva (UTIs) de três hospitais da região Sul na cidade de Porto Alegre, Rio Grande do Sul, de modo a avaliar o perfil de resistência dos principais antibióticos em relação às bactérias identificadas. Em um estudo sobre a prevalência e os resultados de infecções em UTI realizado mundialmente, com a participação de 1.150 centros de 88 países, em que o Brasil foi o terceiro país com maior número de pacientes incluídos, observou-se que a infecção do trato respiratório teve maior prevalência representando $60 \%$ dos casos (Vicent et al., 2020).

Ainda com relação ao sítio de infecção, a segunda mais frequente foi a infecção do trato urinário (ITU). No estudo de Mota, Oliveira e Souto (2018), a urina foi considerada como o material biológico de maior taxa de crescimento microbiano $(49,0 \%)$, este foi um estudo descritivo do tipo retrospectivo, realizado a partir da análise de dados obtidos em 222 prontuários de pacientes internados na UTI do Hospital Santa Casa de Misericórdia de Goiânia. Enquanto, no estudo de Araújo et al. (2018) o crescimento microbiano no trato urinário foi de 40,9\%.

A ITU é definida pela presença de micro-organismo invasor em qualquer região do trato urinário e representa cerca de 30\% a 40\% das IRAS (Mota \& Oliveira, 2019). Quando relacionada à sondagem vesical, sua contaminação pode acontecer durante introdução da sonda, resultante quebra da técnica asséptica; por via ascendente periuretral, através do ambiente extraluminal entre a sonda e a luz da uretra e por contaminação intraluminal (Mota \& Oliveira, 2019).

Dessa forma, a ITU no contexto hospitalar possui um perfil microbiano distinto das comunitárias, comumente estão relacionadas a bactérias gram-negativas como: Pseudomonas aeruginosa, Enterobacter sp., Klebsiella sp.; e algumas grampositivas tal qual: Enterococcus sp., Staphylococcus sp., inclusive fungos do tipo Cândida sp. (Araújo et al., 2018). Na presente pesquisa, a Escherichia coli (100,0\% e 0,0\% nas enfermarias e UTI, respectivamente.) e a Escherichia gergoviae (55,6\% e 44,4\% nas enfermarias e UTI, respectivamente) tiveram uma frequência significativa.

Nesse estudo encontramos resultados importantes de micro-organismos gram-negativos isolados, dentre eles os que mais se destacaram foram Acinetobacter baumannii (53,8\% e 46,2\%, nas enfermarias e UTI, respectivamente) e Pseudomonas aeruginosa (61,5\% nas enfermarias; $38,5 \%$ na UTI). Esse resultado corrobora com o que foi encontrado no estudo de Araújo et al. (2018) no qual o micro-organismo mais isolado foi o Acinetobacter sp. representando 71,05\% das culturas analisadas no estudo citado. Estes resultados corroboram com outras pesquisas, nos quais, foram encontradas incidência de bactérias gramnegativas em $81,1 \%$ na pesquisa de Camponogara e Favarin (2012) e 82\% no estudo de Pereira, Chagas, Freitas, Barros e Caetano, (2016).

As IRAS causadas por bactérias gram-negativas estão relacionadas a perfis de resistência elevados, o que torna 
complexo o estabelecimento do tratamento inicial e têm alto impacto no prognóstico de pacientes críticos, tornando-se responsáveis pelas altas taxas de mortalidade ligadas as IRAS em UTI (Pereira, Chagas, Freitas, Barros \& Caetano, 2016).

Acinetobacter baumannii é considerado um dos principais causadores de infecções graves em pacientes internados, principalmente sepse e pneumonias. Ademais, mostra uma elevada resistência a vários antimicrobianos, e, com isso, os carbapenêmicos se tornaram a principal opção terapêutica, porém, já se observa uma alta taxa de resistência ao imipenem, que se torna cada vez mais ineficaz (Exner et al., 2017). Em nosso estudo, o imipenem teve um perfil de resistência menor (36,0\% e 47,1\%, nas enfermarias e UTI, respectivamente), mas levando em consideração que essa classe de antibiótico é de amplo espectro torna-se uma resistência considerável, quando comparado aos outros antimicrobianos.

Em nossa pesquisa a maior frequência de resistência observada nas enfermarias foi ceftriaxona (84,6\%), seguida de sulfazotrim (83,3\%) e ampicilina com sulbactam (80,0\%). Enquanto na UTI, o maior perfil de resistência foi sulfazotrim $(100,0 \%)$, seguido da ampicilina com sulbactam $(90,0 \%)$, piperacilina com tazabactam sódico $(75,0 \%)$ e ceftriaxona $(72,7 \%)$. Esse perfil de resistência identificado no presente estudo difere do estudo realizado por Gomes et al. (2016) onde a maior resistência foi observada em 85,0\% para meropenem e 81,0\% para imipenem, que teve como objetivo determinar o perfil de suscetibilidade de 60 amostras de Acinetobacter baumannii oriundo de um hospital da cidade do Rio de Janeiro, sendo 27 amostras adquiridas de pacientes de um surto ocorrido em 2011, e um total de 33 amostras obtidas tanto de pacientes quanto do ambiente, coletadas no período de 2014 a 2015, no mesmo hospital. Diante desse perfil, os micro-organismos isolados com essas características resultam em grande impacto clínico, tendo em vista a limitação ou escassez de terapias efetivas no tratamento dessas infecções.

O presente estudo apresentou limitações quanto ao preenchimento adequado das fichas de notificações de IRAS, o que resultou na necessidade de exclusão das mesmas e consequente subnotificação das informações devido à ausência de dados importantes como resultados do antibiograma ou da cultura microbiológica, sítios de infecções e diagnósticos clínicos.

\section{Conclusão}

No presente estudo, o sexo masculino foi o mais acometido por IRAS e a maioria dos pacientes apresentou dois ou mais diagnósticos clínicos, sendo a SIDA, neurotoxoplasmose e tuberculose os mais frequentes. Entre os micro-organismos isolados o que teve maior prevalência foi a Pseudomonas aeruginosa e Acinetobacter baumannii seguido por Escherichia gergoviae. Quanto ao perfil de resistência microbiana, nas enfermarias os resultados mais relevantes foram a cefalosporina de $3^{\text {a }}$ geração, ceftriaxona, seguida de sulfazotrim, enquanto na UTI, as maiores frequências de resistências foram ao sulfazotrim, seguido de ampicilina com sulbactan.

Diante do perfil de resistência encontrado é importante que haja um controle mais eficaz por parte da equipe de saúde para redução e prevenção da disseminação de micro-organismos multirresistentes e o direcionamento da sistematização de condutas que previnam as IRAS. Deste modo, é importante salientar a necessidade do correto preenchimento dos prontuários, pois os dados podem auxiliar a Comissão de Controle de Infecção Hospitalar (CCIH) na tomada de decisões.

Além disso, algumas recomendações para reduzir a incidência das IRAS seriam: vigilância epidemiológica diária do uso dos antibióticos, educação permanente com as equipes multidisciplinares com protocolos rígidos de antibioticoterapia preconizado pela instituição, implantação de barreira na prescrição médica depois do vencimento das doses adequadas para o tratamento, educação permanente com as equipes multidisciplinares para que se tenha uma maior cooperação com a CCIH e Serviço de Controle de Infecção Hospitalar (SCIH) tanto para que o número de subnotificações dos casos diminua, quanto para uma melhora da qualidade do cuidado. Enfatizando a relevância de incentivar o profissional enfermeiro a participar das ações da CCIH e SCIH. 


\section{Referências}

Araújo, B. T. \& Pereira, D. C. R. (2017). Políticas para controle de infecções Relacionadas à Assistência à Saúde (IRAS) no Brasil, Revista Com. Ciências Saúde. 28(3/4), 333-342. https://doi.org/10.51723/ccs.v28i03/04.275http://www.escs.edu.br/revistaccs/index.hp/comumicacaoemcienciasdasaude/article/v iew/275

Araújo, P. L. Mendonça, A. E. O., Medeiros, R. A., Neto, V. L. S., Nobre, T. T. X. \& Costa, I. K. F (2018). Prevalence of health assistance infection in patients hospitalized in intensive therapy unit. Revista Enfermería Global. https://dx.doi.org/10.6018/eglobal.17.4.289311. http://scielo.isciii.es/pdf/eg/v17n52/pt_1695-6141-eg-17-52-278.pdf

Basso, M. E., Pulcinelli, R. S. R., Aquino, A. R. C. \& Santos K. F (2016). Prevalência de infecções bacterianas em pacientes internados em uma unidade de terapia intensiva (UTI). Rev. bras. anal. clin. 48(4). 10.21877/2448-3877.201600307. http://www.rbac.org.br/wp-content/uploads/2017/04/RBAC-vol-48-42016-ref.-307.pdf

Cunha, Q. B. Camponogara, S., Freitas, E. O., Pinno, C., Dias, G. L. \& Cesar, M. P. (2017). Fatores que interferem na adesão às precauções padrão por profissionais de saúde: revisão integrativa. Revista Enfermagem em Foco. 8(1):726. https://doi.org/10.21675/2357-707X.2017.v8.n1.980. http://revista.cofen.gov.br/index.php/enfermagem/article/view/980\#: :text=Ficaram\%20evidentes\%20fatores\%20que\%20influenciam,obst\%C3\%A1 culos\% 20 do\%20trabalho\%3B\%20e\%20fatores

Exner M., Bhattacharya, S., Christiansen, B., Gebel, J., Bermes, P. G., Hartemann, P., Heeg, P. Iischner, C., Kramer, A., Larson, E., Markens, W., Mielke, M., Oltmanns, P., Ross, B., Rotter, M., Schmithausen, R. M., Sonntag, H. G. \& Trautmann, M. (2017). Antibiotic resistance: What issospecial about multidrugresistant Gram-negative bacteria? German Medical Science. Hygiene and Infection Control. 12: https://www.ncbi.nlm.nih.gov/pmc/articles/PMC5388835/

Favarin, S. S. \& Camponogara, S. (2012). Perfil dos pacientes internados na unidade de terapia intensiva adulto de um Hospital Universitário. Revista Enfermagem UFSM. 2(2):320-329. https://doi.org/10.5902/217976925178. https://periodicos.ufsm.br/reufsm/article/view/5178

Ferreira, L. L., Azevedo, L. M. N., Salvador, P. T. C. O., Morais, S. H. M., Paiva, R. M. \& Santos, V. E. P. (2019). Nursing Care in Healthcare-Associated Infections: A Scoping Review Cuidado de enfermería en las Infecciones Relacionadas con la Asistencia Sanitaria: Scoping review. Revista Brasileira

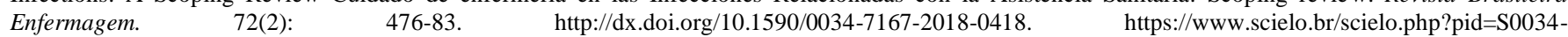
$71672019000200476 \&$ script $=$ sci_abstract

Gomes, D. B. C., Genteluci, G. L., Carvalho, K. R., Medeiros, L. M., Almeida, V. C., Castro, E. A. R. \& Boas, M. H. S. V. (2016). Multidrug Resistant Acinetobacer baumanni: the occurrence of polymyxin resistance in Rio de Janeiro. Revista Visa em Debate. 4(3):28-34. https://doi.org/10.22239/2317269x.00732. https://visaemdebate.incqs.fiocruz.br/index.php/visaemdebate/article/view/732

Mota, F. S., Oliveira, H. A. \& Souto, R. C. F. (2018). Hot dog marketed on public roads: microbiological and hygienic sanitary analysis. Brazilian Journal of Clinical Analyes. Goiânia-GO. 10.21877/2448-3877.201800740. http://www.rbac.org.br/artigos/perfil-e-prevalencia-de-resistencia-aos-antimicrobianos-debacterias-gram-negativas-isoladas-de-pacientes-de-uma-unidade-de-terapia-intensiva/

Nere, C. S., Silva, N. B., Silva, N. M. S., Rodrigues, T. E. S., Pereira, E. S. \& Soares, J. L. (2017). The performance of nursing in the hospital infection: Integrative review. Revista ciências \& Saberes. FACEMA, Maranhão. https://doi.org/10.1590/0034-7167-2018-0418. https://www.scielo.br/scielo.php?pid=S0034-71672019000200476\&script=sci_abstract

Pereira, F. G. F., Chagas, A. N. S., Freitas, M. M. C., Barros, L. M. \& Caetano, J. Á. (2016). Caracterização das infecções relacionadas à assistência à saúde em uma Unidade de Terapia Intensiva. Revista Visa em Debate. Vigilância Sanitária em Debate: Sociedade, Ciência \& Tecnologia, 4(1), 70-77. https://doi.org/10.3395/2317-269x.00614. https://visaemdebate.incqs.fiocruz.br/index.php/visaemdebate/article/view/614

Prates, F. I. F., Silva, G. F., Fernandes, R. A. \& Cesar, J. J. (2020). Damages Caused By Bacterial Resistance: A World Public Health Problem. Brazilian Journal of Surgery and Clinical Research - BJSCR, 32(2), 131-138. https://www.mastereditora.com.br/periodico/20201004_093315.pdf

Santos, A. V., Silva, M. R. P., Carvalho, M. M., Carvalho, L. R. B., Moura, M. E. B. \& Landim, C. A. P. (2016). Perfil das infecções hospitalares nas unidades de terapia intensiva de um hospital de urgência. Revista de Enfermagem UFPE On-Line, 10(1), 194-201. 10.5205/reuol.7901-80479-1. https://periodicos.ufpe.br/revistas/\%20revistaenfermagem/article/viewFile/10940/12241

Silva, P. S., Silva, T. R., Hoyashi, C. M. T. \& Pereira, R. M. S. (2017). Prevenção e controle de infecções relacionadas à assistência à saúde: fatores extrínsecos ao paciente. HU Revista, Juiz de Fora, 43(3), 277-283. https://doi.org/10.34019/1982-8047.2017.v43.2739. https://periodicos.ufjf.br/index.php/hurevista/article/view/2739

Vincent, J. L., Sakr, Y., Singer, M., Loeches, I. M., Machado, F. R., Marshall, J. C, Finfer, S. Pelosi, P., Brazzi, L., Aditianingsih, D., Timsit, J. F., Du, B., Wittebole, X., Máca, J., Kannan, S., Delsol, L. A. G., Waele, J. J. D., Mehta, Y., Bonten, M. J. M., Khanna, A. K., Kollef, M., Human, M., Angus, D. C. (2020). Prevalence and Outcomes of Infection Among Patients in Intensive Care Units in 2017. JAMA. 323(15):1478-1487. 10.1001/jama.2020.2717. https://jamanetwork.com/journals/jama/fullarticle/2763669

World Health Organization (2015). Worldwide country situationanalysis: response toantimicrobialresis-tance. WHO Press. http://www.who.int/drugresistance/documents/situationanalysis/en/ 